\title{
Predictive value of radiological features on spread through air space in stage cIA lung adenocarcinoma
}

\author{
Zhenrong Zhang ${ }^{1}$, Zhan Liu ${ }^{2}$, Hongxiang Feng ${ }^{1}$, Fei Xiao ${ }^{1}$, Weipeng Shao ${ }^{2}$, Chaoyang Liang ${ }^{1}$, \\ Hongliang Sun ${ }^{3}$, Xinlei Gu${ }^{4}$, Deruo Liu ${ }^{1}$ \\ ${ }^{1}$ Department of General Thoracic Surgery, China-Japan Friendship Hospital, Beijing, China; ${ }^{2}$ Department of General Thoracic Surgery, China- \\ Japan Friendship Hospital, Peking University China-Japan Friendship School of Clinical Medicine, Beijing, China; ${ }^{3}$ Department of Radiology, \\ China-Japan Friendship Hospital, Beijing, China; ${ }^{4}$ Department of Thoracic Surgery, Peking University International Hospital, Beijing, China \\ Contributions: (I) Conception and design: Z Zhang, D Liu; (II) Administrative support: Z Zhang, C Liang, D Liu; (III) Provision of study materials \\ or patients: Z Zhang, H Feng, F Xiao, H Sun; (IV) Collection and assembly of data: Z Zhang, Z Liu, W Shao, H Sun, X Gu; (V) Data analysis and \\ interpretation: Z Zhang, Z Liu; (VI) Manuscript writing: All authors; (VII) Final approval of manuscript: All authors. \\ Correspondence to: Zhenrong Zhang; Deruo Liu. Department of General Thoracic Surgery, China-Japan Friendship Hospital, No. 2 Yinghua East \\ Road, Chaoyang District, Beijing 100029, China. Email: doctor_zzr@126.com; deruoliu@vip.sina.com.
}

Background: Spread through air space (STAS) is a risk factor for disease recurrence in patients with stage IA lung adenocarcinoma (LUAD) who undergo limited resection. Preoperative prediction of STAS could help intraoperative surgical decision-making in small LUAD patients. The aim of the study was to evaluate the predictive value of radiological features on STAS in stage cIA LUAD.

Methods: A case-control study was designed through retrospective analysis of the radiological features of patients who underwent curative surgery for LUAD with a clinical tumor size $\leq 3 \mathrm{~cm}$. Univariable and multivariable analyses were used to identify the independent risk factors for STAS. The predicted probability of STAS was calculated by a specific formula. Receiver operating characteristic (ROC) curves were used to determine a cut-off value with appropriate specificity while maintaining high sensitivity for STAS positivity.

Results: STAS was frequently observed in acinar predominant $(\mathrm{P}<0.001)$, micropapillary predominant $(\mathrm{P}<0.001)$ and solid predominant $(\mathrm{P}<0.001)$ tumors and was significantly associated with larger $\mathrm{pT}$ size $(\mathrm{P}<0.001)$, presence of micropapillary component $(\mathrm{P}<0.001)$, lymphovascular invasion (LVI) $(\mathrm{P}<0.001)$, visceral pleura invasion (VPI) $(\mathrm{P}<0.001)$, both $\mathrm{N} 1$ and $\mathrm{N} 2$ lymph node metastasis $(\mathrm{P}<0.001)$ and ALK rearrangement $(\mathrm{P}<0.001)$. STAS-positivity was significantly associated with the presence of cavitation $(\mathrm{P}=0.047)$, lobulation $(\mathrm{P}=0.009)$, air bronchogram $(\mathrm{P}<0.001)$, and vascular convergence $(\mathrm{P}=0.016)$ and was also associated with greater maximum tumor diameter $(\mathrm{P}<0.001)$, maximum solid component diameter $(\mathrm{P}<0.001)$, maximum tumor area $(\mathrm{P}<0.001)$, consolidation/tumor ratio $(\mathrm{CTR})(\mathrm{P}<0.001)$, tumor disappearance ratio (TDR) $(\mathrm{P}<0.001)$ and computed tomography $(\mathrm{CT})$ value $(\mathrm{P}<0.001)$. Multivariable analysis showed that STAS was associated with air bronchogram $(\mathrm{P}=0.042)$, maximum tumor diameter $(\mathrm{P}=0.015)$, maximum solid component diameter $(\mathrm{P}=0.022)$ and $\mathrm{CTR}(\mathrm{P}<0.001)$. The ROC curve showed that the area under the curve (AUC) was 0.726 in the model for predicting STAS, with a sensitivity and specificity of $95.2 \%$ and $36.8 \%$, respectively.

Conclusions: STAS-positive LUAD was associated with air bronchogram, maximum tumor diameter, maximum solid component diameter and CTR. These radiological features could predict STAS with excellent sensitivity but inferior specificity.

Keywords: Radiology; spread through air space (STAS); lung adenocarcinoma (LUAD)

Submitted May 01, 2020. Accepted for publication Oct 10, 2020.

doi: $10.21037 /$ jtd-20-1820

View this article at: http://dx.doi.org/10.21037/jtd-20-1820

(c) Journal of Thoracic Disease. All rights reserved. 


\section{Introduction}

Lung cancer has been reported as the leading cause of cancer morbidity and mortality worldwide $(1,2)$, and lung adenocarcinoma (LUAD) is the most common histological type of lung cancer (2). With the discovery that low-dose computed tomography (LDCT) screening could lead to a reduction of $20 \%$ mortality in high-risk patients (3), worldwide implementation of computed tomography (CT) has resulted in a drastic increase in the detection of small lung cancer patients, especially those presenting with ground glass nodules (GGNs) (4).

Lobectomy has been accepted as the standard surgical procedure in early-stage lung cancer after the Lung Cancer Study Group showed a preference for lobectomy rather than sublobar resection (5). Several studies have reported favorable results with sublobectomy in GGN-predominant patients (6-8). Two ongoing randomized, phase III trials (JCOG0802/WJOG4607L and CALGB140503) of lobectomy versus limited resection (i.e., segmentectomy) for small lung cancer will provide updated robust evidence regarding the preferred surgical procedures for invasive peripheral lung cancers (predominantly solid predominant nodules on chest CT) $(9,10)$.

In 2015, the World Health Organization (WHO) classification of lung cancer proposed the concept of spread through air spaces (STAS) as a new indicator of the pattern of invasion in LUAD (11). Since then, many studies have reported that STAS is a risk factor for disease recurrence in patients with stage I LUAD who underwent limited resection $(12,13)$. It was also associated with significantly reduced overall and recurrence-free survival (14). It was suggested that lobectomy should still be preferred if STAS was recognized, and postoperative chemotherapy should be considered in these cases (12). The prediction of STAS preoperatively without histologic confirmation could help intraoperative surgical decision-making in small LUAD patients, especially in those with radiological features such as GGN.

The objective of this retrospective study was to investigate the association between radiological features and STAS. We present the following article in accordance with the STROBE reporting checklist (available at http://dx.doi. org/10.21037/jtd-20-1820).

\section{Methods}

A retrospective case-control study was carried out between January 2017 and December 2018 in a single academic thoracic surgery center at the China-Japan Friendship Hospital, Beijing, China. The study was conducted in accordance with the Declaration of Helsinki (as revised in 2013). The study was approved by the institutional ethics board of China-Japan Friendship Hospital (No. 2018-13K08), and individual consent for this retrospective analysis was waived.

\section{Study population}

As a result, 762 consecutive patients who underwent curative surgery for LUAD with a clinical tumor size $\leq 3 \mathrm{~cm}$ were enrolled. Only clinical T1a-cN0M0 patients were included. Patients were excluded from the study cohort if one of the following criteria was met: history of previous lung operation, other histological type such as squamous cell carcinoma or small cell lung cancer, metastatic LUAD, multiple lung nodules, specific variants of adenocarcinoma such as fetal or enteric adenocarcinoma, or previous neoadjuvant antitumor therapy. All patients were staged according to the eighth edition of the tumor-nodemetastasis classification proposed by the IASLC Lung Cancer Staging Project (15).

\section{Clinicopathological characteristics}

All patients' medical records and postoperative pathology reports were reviewed. The clinicopathological characteristics, including age at surgery, sex, smoking status, preoperative serum carcinoembryonic antigen (CEA) level, type of surgical procedure, histological subtype (WHO classification 2015), tumor size, lymph node metastasis, pathologic tumor-node-metastasis stage, visceral pleura invasion (VPI), lymphovascular invasion (LVI) and genetic alteration information, were examined. Two experienced pathologists who were blinded to the patients' clinical outcomes reviewed the hematoxylin and eosin (H\&E)-stained tissue sections. Consensus was reached through discussion in cases of disagreement; otherwise, a senior pathologist was required to re-evaluate the CT images. According to the 2015 WHO classification of lung cancer, STAS was defined as pathological micropapillary clusters separated from the main tumor, solid nests separated from the main tumor or single cells beyond the edge of the tumor (11).

\section{Radiological characteristics}

Radiological data including tumor location (left or right 


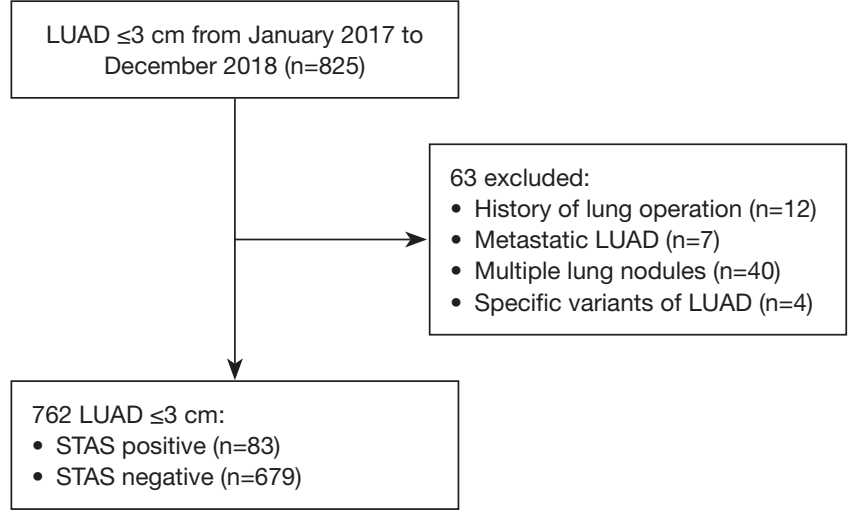

Figure 1 Flow chart for the study population. LUAD, lung adenocarcinoma; STAS, spread through air space.

side, peripheral or central), maximum tumor diameter on the lung window, maximum solid component diameter on the lung window, consolidation/tumor ratio (CTR), maximum tumor area on the lung window, maximum tumor diameter on the mediastinal window, tumor disappearance ratio (TDR) and CT value on high-resolution computed tomography (HRCT) were collected. Other CT features, such as notch, spiculation, pleural indentation, cavitation, vascular convergence and air bronchogram, were also evaluated.

All enrolled patients underwent HRCT within 2 weeks preoperatively. HRCT was performed with the patient in the supine position during inspiratory breath-hold using various multidetector row scanners: Aqui-lion 4 (TOSHIBA Corporation, Tokyo, Japan), SOMA-TOM Plus4 Volume Zoom (SIEMENS, Munich, Germany), Brilliance CT (Philips, Amsterdam, the Netherlands). The imaging parameters were as follows: tube voltage, $120 \mathrm{kVp}$; tube current, 100-150 mA; detector collimation, $0.625-1.5 \mathrm{~mm}$; beam pitch, 1.375-1.5.

Two experienced radiologists who were blinded to the patients' clinicopathological features reviewed the chest CT images independently. Consensus was reached through discussion in case of disagreement; otherwise, a senior radiologist was required to re-evaluate the $\mathrm{CT}$ images. The measurement data were all measured on the crosssectional images of the chest $\mathrm{CT}$ in picture archiving and communication systems (PACS) (lung window setting: width, 1,500 $\mathrm{HU}$ and level, $-600 \mathrm{HU}$; mediastinal window setting: width, $350 \mathrm{HU}$ and level, $40 \mathrm{HU}$ ) three times, and the average value was taken. The Auto Contour software in the workstation was used to draw the boundaries of the lesions on the CT images of the lung window and the mediastinal window. During the measurement, the influence of calcification, blood vessels, bronchi, and volume effect were avoided as much as possible, and then the maximum area and the CT value of the lesions were automatically generated by the computer. The CTR was defined as the proportion of the maximum consolidation (C) diameter divided by the maximum tumor $(\mathrm{T})$ diameter on the lung window. The TDR was defined as $(1-$ the maximum crosssectional area on mediastinal window/the maximum crosssectional area on lung window) $\times 100 \%$.

\section{Results}

\section{Patient characteristics}

From January 2017 to December 2018, 825 patients who underwent surgery for LUAD with a clinical tumor size $\leq 3 \mathrm{~cm}$ were enrolled; 63 patients were excluded from the study cohort because of a history of previous lung operation $(\mathrm{n}=12)$, metastatic LUAD ( $\mathrm{n}=7)$, multiple lung nodules $(n=40)$, or specific variants of adenocarcinoma, such as fetal or enteric adenocarcinoma $(n=4)$. After exclusion, 762 patients (83 STAS-positive patients and 679 STAS-negative patients) were enrolled in this study (Figure 1).

The patients' baseline characteristics are summarized in Table 1. There were 276 men and 486 women with an average age of 59 years old in the cohort. A total of 595 patients underwent lobectomy, and sublobectomy (47 segmentectomies and 120 wedge resections) was performed in 167 patients. Lymph node dissection was performed in 664 patients, and 98 patients did not undergo lymph node dissection. This study enrolled 645 patients with invasive LUAD, 97 patients with microinvasive adenocarcinoma (MIA) and 20 patients with adenocarcinoma in situ (AIS). Of the invasive LUADs, most cases were acinar predominant (361, 47.4\%), followed by lepidic predominant (163, 21.4\%) and papillary predominant (40,5.2\%). Micropapillary components were pathologically detected in 161 cases. N1 and N2 lymph node involvement occurred in 63 and 52 cases, respectively. A total of 584 patients received molecular tests, such as EGFR, ALK and ROS1. A total of 343 (343/584) patients harbored EGFR mutations, 11 (11/501) had ALK rearrangement and 5(5/500) had ROS1 rearrangement.

\section{STAS correlation with clinicopathological characteristics}

STAS was frequently observed in acinar predominant 
Table 1 Patient characteristics

\begin{tabular}{|c|c|}
\hline Patient characteristic & Data, n (\%) \\
\hline Number of patients & 762 \\
\hline Age (years) & $59 \pm 10$ \\
\hline \multicolumn{2}{|l|}{ Gender } \\
\hline Male & 276 (36.2) \\
\hline Female & $486(63.8)$ \\
\hline \multicolumn{2}{|l|}{ Smoke status } \\
\hline Smoker & $137(18.0)$ \\
\hline Non-smoker & $625(82.0)$ \\
\hline \multicolumn{2}{|l|}{ CEA (ng/mL) } \\
\hline$<5$ & $660(86.6)$ \\
\hline$\geq 5$ & $76(10.0)$ \\
\hline \multicolumn{2}{|l|}{ Operation } \\
\hline Lobectomy & $595(78.1)$ \\
\hline Segmentectomy & $47(6.2)$ \\
\hline Wedge resection & $120(15.7)$ \\
\hline \multicolumn{2}{|l|}{ Tumor location } \\
\hline Left & 337 (44.2) \\
\hline Right & $425(55.8)$ \\
\hline Upper lobe & $483(63.4)$ \\
\hline Middle lobe & $51(6.7)$ \\
\hline Lower lobe & 228 (29.9) \\
\hline Peripheral & $744(97.6)$ \\
\hline Central & $18(2.4)$ \\
\hline \multicolumn{2}{|l|}{ CT features } \\
\hline Cavitation & $66(8.7)$ \\
\hline Lobulation & $551(72.3)$ \\
\hline Spiculation & $476(62.5)$ \\
\hline Pleura indentation & $371(48.7)$ \\
\hline Air bronchogram & $92(12.1)$ \\
\hline Vascular convergence & $687(90.2)$ \\
\hline Maximum tumor diameter (mm) & $15.03(11.00,20.14)$ \\
\hline $\begin{array}{l}\text { Maximum solid component } \\
\text { diameter }(\mathrm{mm})\end{array}$ & $6.35(6.35,15.70)$ \\
\hline $\begin{array}{l}\text { Maximum area on lung window } \\
\left(\mathrm{mm}^{2}\right)\end{array}$ & $99.02(49.98,175.68)$ \\
\hline CTR & $0.45(0,0.92)$ \\
\hline
\end{tabular}

Table 1 (continued)
Table 1 (continued)

\begin{tabular}{|c|c|}
\hline Patient characteristic & Data, n (\%) \\
\hline TDR & $0.99(0.41,1.00)$ \\
\hline CT value $(\mathrm{Hu})$ & $-363.50(-582.25,-56.75)$ \\
\hline \multicolumn{2}{|l|}{ Histological subtype } \\
\hline AIS & $20(2.6)$ \\
\hline MIA & $97(12.7)$ \\
\hline Lepidic predominant & $163(21.4)$ \\
\hline Acinar predominant & $361(47.4)$ \\
\hline Papillary predominant & $40(5.2)$ \\
\hline Micropapillary predominant & $8(1.0)$ \\
\hline Solid predominant & $34(4.5)$ \\
\hline Mucinous type & $20(2.6)$ \\
\hline Unclassified type & $19(2.5)$ \\
\hline Micropapillary component & $161(21.1)$ \\
\hline pT size $(\mathrm{cm})$ & $1.40(1.00,2.00)$ \\
\hline N1 & $63(8.3)$ \\
\hline N2 & $52(6.8)$ \\
\hline VPI & $164(21.5)$ \\
\hline LVI & $26(3.4)$ \\
\hline STAS & $83(10.9)$ \\
\hline EGFR mutation & $343(45.0)$ \\
\hline ALK rearrangement & $11(1.4)$ \\
\hline ROSI rearrangement & $5(0.7)$ \\
\hline
\end{tabular}

CEA, carcinoembryonic antigen; CT, computed tomography; CTR, consolidation/tumor ratio; TDR, tumor disappearance ratio; AIS, adenocarcinoma in situ; MIA, microinvasive adenocarcinoma; VPI, visceral pleura invasion; LVI, lymphovascular invasion; STAS, spread through air space.

$(\mathrm{P}<0.001)$, micropapillary predominant $(\mathrm{P}<0.001)$, and solid predominant $(\mathrm{P}<0.001)$ tumors. STAS was also significantly associated with larger $\mathrm{pT}$ size $(\mathrm{P}<0.001)$, presence of a micropapillary component $(\mathrm{P}<0.001)$, LVI $(\mathrm{P}<0.001)$, visceral pleural invasion $(\mathrm{P}<0.001)$ and both $\mathrm{N} 1$ and $\mathrm{N} 2$ lymph node metastasis $(\mathrm{P}<0.001)$. STAS was frequently observed in tumors with ALK rearrangement $(\mathrm{P}<0.001)$ but not in tumors with EGFR mutations $(\mathrm{P}=0.376)$. Furthermore, the characteristics of age, sex, CEA level, smoking status and type of surgical procedure were not significantly associated with STAS (Table 2). 
Table 2 STAS with clinicopathological characteristics

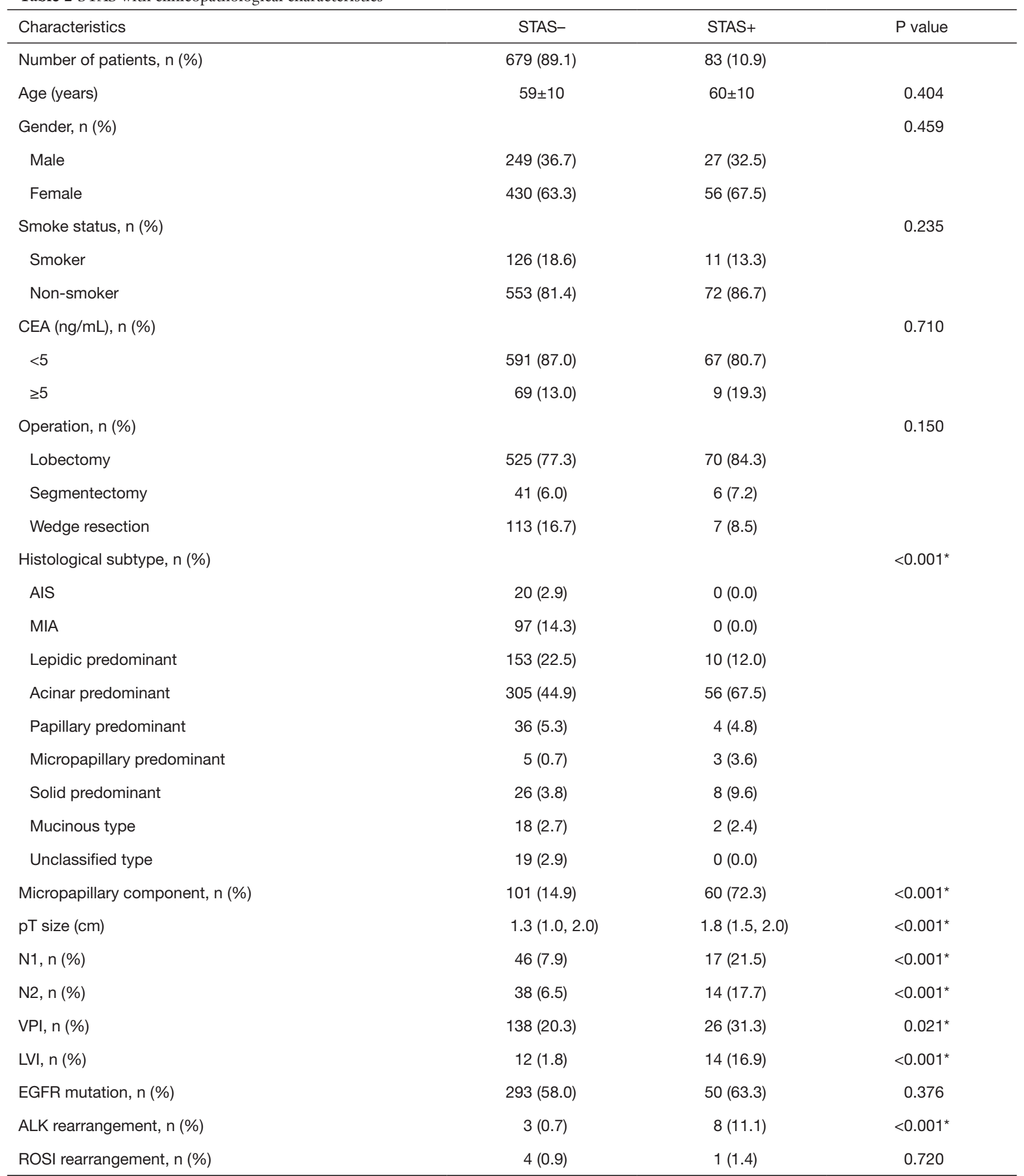

*, the results were significantly different, which means the $P$ value is less than 0.05 . STAS, spread through air space; CEA, carcinoembryonic antigen; AIS, adenocarcinoma in situ; MIA, microinvasive adenocarcinoma; VPI, visceral pleura invasion; LVI, lymphovascular invasion. 
Table 3 STAS with radiological features

\begin{tabular}{|c|c|c|c|}
\hline Characteristics & STAS- & STAS+ & $P$ value \\
\hline \multicolumn{4}{|l|}{ Tumor location, n (\%) } \\
\hline Left & $297(43.7)$ & $40(48.2)$ & 0.441 \\
\hline Right & $382(56.3)$ & $43(51.8)$ & \\
\hline Middle & $46(6.8)$ & $5(6.0)$ & \\
\hline Lower & $195(28.7)$ & $33(39.8)$ & \\
\hline Peripheral & $664(97.8)$ & $80(96.4)$ & 0.426 \\
\hline Central & $15(2.2)$ & $3(3.6)$ & \\
\hline Lobulation & $481(70.8)$ & $70(84.3)$ & $0.009^{*}$ \\
\hline Spiculation & $420(61.9)$ & $56(67.5)$ & 0.319 \\
\hline Pleura indentation & $324(47.7)$ & $47(56.6)$ & 0.125 \\
\hline Air bronchogram & $72(10.6)$ & $20(24.1)$ & $<0.001^{*}$ \\
\hline Vascular convergence & $606(89.2)$ & $81(97.6)$ & $0.016^{\star}$ \\
\hline Maximum tumor diameter (mm) & $14.9(10.7,20.0)$ & $18.1(15.0,21.2)$ & $<0.001^{*}$ \\
\hline Maximum solid component diameter (mm) & $5.0(0,14.7)$ & $15.0(7.9,19.1)$ & $<0.001^{*}$ \\
\hline Maximum tumor area $\left(\mathrm{mm}^{2}\right)$ & $94.5(45.1,168.8)$ & $136.8(81.8,191.4)$ & $<0.001^{*}$ \\
\hline
\end{tabular}

*, the results were significantly different, which means the $P$ value is less than 0.05 . STAS, spread through air space; CT, computed tomography; CTR, consolidation/tumor ratio; TDR, tumor disappearance ratio.

\section{STAS with radiological features}

Tumor STAS was observed in 83 cases (10.89\%). STASpositivity was significantly associated with the presence of several radiological features, including cavitation $(\mathrm{P}=0.047)$, lobulation $(\mathrm{P}=0.009)$, air bronchogram $(\mathrm{P}<0.001)$ and vascular convergence $(\mathrm{P}=0.016)$ (Table 3). STAS was also associated with greater maximum tumor diameter $(\mathrm{P}<0.001)$, maximum solid component diameter $(\mathrm{P}<0.001)$, cross-sectional area on the lung window $(\mathrm{P}<0.001)$, CTR $(\mathrm{P}<0.001)$, TDR $(\mathrm{P}<0.001)$ and $\mathrm{CT}$ value $(\mathrm{P}<0.001)$ (Figure 2$)$.

\section{Logistic regression analysis and ROC curve}

To predict STAS status with preoperative radiological features to help surgical decision-making intraoperatively, logistic regression analysis was first performed to detect risk factors for STAS. Multivariable analysis showed that STAS was associated with air bronchogram $(\mathrm{OR}=1.85,95 \% \mathrm{CI}$ : 1.02-3.34, $\mathrm{P}=0.042)$, maximum tumor diameter $(\mathrm{OR}=1.11$, 95\% CI: $1.02-1.22, \mathrm{P}=0.015)$, maximum solid component diameter $(\mathrm{OR}=0.88,95 \% \mathrm{CI}: 0.79-0.98, \mathrm{P}=0.022)$ and CTR $(\mathrm{OR}=48.73$, 95\% CI: 6.23-381.19, $\mathrm{P}<0.001)$ (Table 4). The Hosmer and Lemeshow test showed a $\mathrm{P}$ value of 0.515 $(>0.05)$. The model coefficient $\mathrm{P}$ value was less than 0.001 in the omnibus tests. The predicted probability of STAS can be calculated by the following formula:

$\operatorname{Logit}(\mathrm{P})=-4.944+0.615 \times$ air bronchogram +0.108 $\times$ maximum tumor diameter $-0.130 \times$ maximum solid 

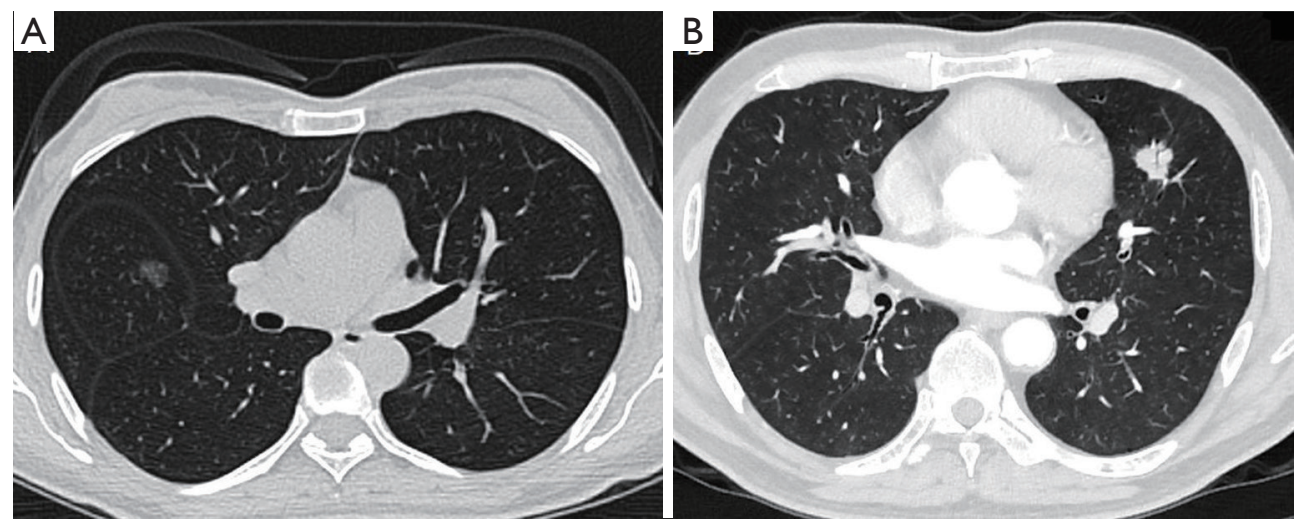

Figure 2 Representative CT images of the cases with and without STAS. (A) A 45-year-old female patient pathologically confirmed as LUAD with STAS-negative features. Chest CT showed a pure GGO in the middle lobe of the right lung; the maximum tumor diameter was $13 \mathrm{~mm}$, and the CTR was 0; (B) a 59-year-old male patient with pathologically confirmed LUAD with STAS-positive features. Chest CT showed a solid nodule with air bronchogram in the upper lobe of the left lung. The maximum tumor diameter was 17 mm, and the CTR was 1. CT, computed tomography; STAS, spread through air space; LUAD, lung adenocarcinoma; GGO, ground glass opacity; CTR, consolidation/tumor ratio.

Table 4 Multivariable analysis for the relationship between STAS and radiological features

\begin{tabular}{lcc}
\hline Characteristics & OR $(95 \% \mathrm{Cl})$ & $\mathrm{P}$ value \\
\hline Air bronchogram & & 0.042 \\
- & 1.00 & \\
+ & $1.85(1.02-3.34)$ & 0.015 \\
Maximum tumor diameter $(\mathrm{mm})$ & $1.11(1.02-1.22)$ & 0.022 \\
Maximum solid component diameter $(\mathrm{mm})$ & $0.88(0.79-0.98)$ & $<0.001$ \\
CTR & $48.73(6.23-381.19)$ & \\
\hline
\end{tabular}

STAS, spread through air space; CTR, consolidation/tumor ratio.

component diameter $+3.886 \times$ CTR .

$\mathrm{P}=\mathrm{e}^{\operatorname{Logit}(\mathrm{P})} /\left[1+\mathrm{e}^{\operatorname{Logit}(\mathrm{P})}\right]$.

ROC curves were used to determine the cut-off value that had appropriate specificity while maintaining high sensitivity for STAS positivity with the predicted probability generated by the formula above. The AUC was 0.726 with a $\mathrm{P}$ value of less than 0.001 . The Youden index was 0.320 , and the sensitivity and specificity were $95.2 \%$ and $36.8 \%$, respectively (Figure 3).

The association between STAS status and histological and genetic features was also analyzed with logistic regression analysis. STAS commonly presented with the presence of micropapillary components (OR $=13.88,95 \%$ CI: 7.28-26.47, $\mathrm{P}<0.001)$, LVI (OR =9.06, 95\% CI: $2.40-$ $34.17, \mathrm{P}<0.001)$ and $\mathrm{ALK}$ rearrangement $(\mathrm{OR}=8.46,95 \%$
CI: 1.46-49.26, P=0.009) (Table 5). There was no association between STAS status and other histological and genetic features, including visceral pleural invasion $(\mathrm{P}=0.056), \mathrm{pT}$ size $(\mathrm{P}=0.055), \mathrm{EGFR}(\mathrm{P}=0.404)$ and $\operatorname{ROS} 1(\mathrm{P}=0.859)$.

\section{Discussion}

STAS is a new indicator of invasion in LUAD proposed by the WHO in 2015; STAS features include pathological micropapillary clusters separated from the main tumor, solid nests separated from the main tumor or single cells beyond the edge of the tumor (11). As low-dose chest CT and HRCT are widely accepted, small lung cancers, especially lung cancers with GGN features, are increasingly being detected. Many studies have shown satisfactory 
outcomes after limited resection, such as segmentectomy or wedge resection (16-18). The National Comprehensive Cancer Network (NCCN) guidelines announced that sublobectomy could be an alternative to lobectomy in lung cancer patients when they meet at least one of the following criteria: pathologically confirmed AIS, GGN\% $>50 \%$ and radiological tumor doubling time $\geq 400$ days (19). The two largest ongoing clinical trials verified the long-term

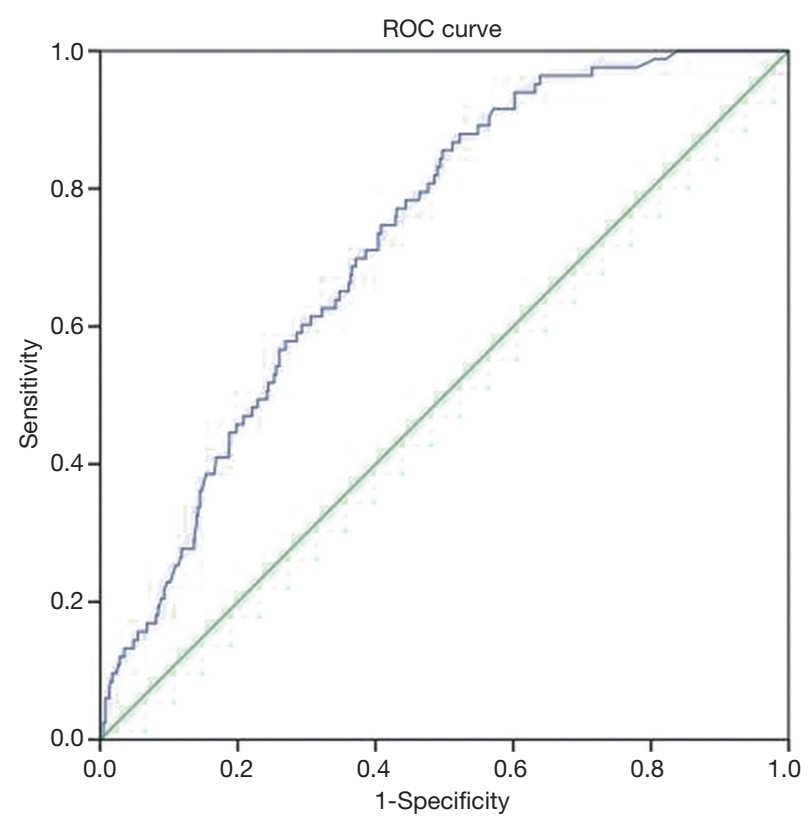

Figure 3 ROC curve shows that the AUC of the model for predicting STAS was 0.726 . ROC, receiver operating characteristic; AUC, area under the curve; STAS, spread through air space. outcome of sublobectomy compared with lobectomy $(9,10)$.

Recently, a growing number of studies have reported STAS as a significant risk factor for prognosis in LUAD $(13,20)$. Masai et al. reported that the presence of STAS is a significant risk factor for local recurrence in early-stage lung cancer after limited resection (13). Similarly, in Kadota and colleagues' study, they observed that in patients with stage I LUAD with STAS, limited resection was associated with a higher risk of recurrence. However, in those without STAS, there was no significant difference in the risk of recurrence between lobectomy and limited resection (20). Therefore, the authors suggested that lobectomy should remain the standard treatment option for early-stage LUAD patients with STAS, and if findings suggestive of STAS are observed, additional resection, including conversion to lobectomy, should be considered for good-risk patients $(13,20)$. However, most of the studies are retrospective studies at present, and further prospective studies are needed to identify the effect of STAS on surgical procedures in patients with stage IA LUAD.

Considering that STAS-positive patients who underwent limited resection had higher risks of recurrence and the presence of STAS suggests the presence of a potential positive surgical margin under limited resection (21), it is worth predicting the presence of STAS to help surgical decision-making preoperatively. Several studies have tried to predict STAS status with specific pathological features (22-24). However, highly experienced pathologists should be requested if precise intraoperative frozen diagnosis is needed. Therefore, to the methods for how to predict STAS using preoperative radiological features could have a

Table 5 Multivariable analysis for the relationship between STAS and histological and genetic features

\begin{tabular}{lcc}
\hline Characteristics & OR $(95 \%$ Cl) & P value \\
\hline Micropapillary component & 1.00 & $<0.001$ \\
- & $13.88(7.28-26.47)$ & 0.009 \\
+ & & 1.00 \\
ALK arrangement & $8.46(1.46-49.26)$ & $<0.001$ \\
- & & 1.00 \\
+ & $9.06(2.40-34.17)$ & \\
+ & & \\
\hline
\end{tabular}

STAS, spread through air space; LVI, lymphovascular invasion. 
significant clinical impact.

Previous studies have suggested that STAS could be associated with several radiologic features (22,25-27). Toyokawa $e t a l$. demonstrated that STAS was significantly associated with larger radiologic tumor diameter $(\mathrm{P}=0.008)$ and higher CTR $(\mathrm{P}<0.001)(22)$. Similarly, Kim and colleagues observed that the maximum diameter of the solid component, the presence of vascular convergence and the absence of air bronchogram were associated with STAS (25). With 726 patients under analysis, our results similarly showed that the presence of STAS was associated with maximum tumor diameter, maximum solid component diameter and CTR. In contrast to Kim and colleagues' study, our study observed that the presence of air bronchograms was associated with STAS. Both univariate and multivariate analyses confirmed these results.

Different results have been achieved in terms of the association between the GGN component and STAS. Additionally, in Kim and colleagues' study, no pure GGN was observed to be STAS positive (25). In other studies, STAS was pathologically confirmed in $5-11 \%$ pure GGN patients $(26,27)$. In our study, STAS was observed in $2.42 \%$ (7/289) of pure GGN patients and in $10.62 \%$ (12/113) of GGN-predominant patients, which means that the choice of sublobectomy or lobectomy should be made not only on the presence of pure GGN but also on the combination of several radiological features. By combining radiological features that were associated with STAS, we tried to calculate the predicted probability of STAS in each patient. A ROC curve with an AUC of 0.726 was built; it had excellent sensitivity (95.2\%) but inferior specificity (36.8\%).

The features most often considered were the correlation between pathological characteristics and STAS. Toyokawa $e t$ al. observed that the pT size of the lesion was significantly associated with STAS. By analyzing a cohort of adenocarcinomas, their study demonstrated distinct correlations between STAS and larger tumor size $(\mathrm{P}=0.004)$, pleural invasion $(\mathrm{P}=0.027)$ and invasive behavior $(\mathrm{P}<0.001)(22)$. Our study showed that both a higher maximum tumor diameter and maximum solid component diameter were associated with the presence of STAS. The odds ratio of the CT finding "maximum solid component diameter" was 0.88 $(0.79-0.98)$ by multivariable analysis for the relationship between STAS and radiological features. This odds ratio showed that the maximum solid component diameter had a negative correlation with the presence of STAS. A correlation tendency was also observed with pathological tumor size $(\mathrm{P}=0.055)$.
Recent studies have shown that LUAD with the presence of a micropapillary pattern is considered to have a more invasive mechanism $(28,29)$. In a study presented by Lee and colleagues, the incidence of STAS was significantly higher in LUAD with micropapillary patterns, and STAS was suggested as a potential parameter for tumor aggressiveness (23). Similar results were observed in our study, and an obvious connection between micropapillary pattern and STAS was confirmed. Our study also found that STAS could also be accompanied by LVI, which was consistent with other studies (24). In this study, we found that STAS had a significant association with LVI. These results showed that STAS may be one of the invasive mechanisms in LUAD with a micropapillary pattern and in LVI.

The correlation between genetic alteration and STAS was further analyzed in our study. Consistent with previous reported studies $(14,20,23)$, STAS was confirmed to be strongly associated with ALK expression but negatively associated with EGFR mutation. In the abovementioned study (23), STAS was significantly associated with the absence of wild-type EGFR rather than EGFR mutations. In Warth and colleagues' study, after analyzing 569 resected LUAD patients, STAS was associated with lower rates of EGFR mutation. In Kadota and colleagues' study, STAS was more frequently identified in ALK-positive patients $(\mathrm{P}=0.020)$ than in ALK-negative patients (20).

This study has several limitations. First, this study was limited by its retrospective design. Second, all the included parameters, both radiological and pathological, could be influenced by subjectivity. Third, with this model, the predicted real positive rate (sensitivity) was acceptable (95.2\%), but the predicted true negative rate was only $36.8 \%$, which means that this model should be interpreted cautiously in the selection of candidates for limited resection, and this model should be verified in a larger population.

In conclusion, we verified that STAS positivity was commonly present in LUAD patients with micropapillary components, LVI and ALK rearrangement. In addition, we confirmed that STAS-positive LUAD was associated with air bronchogram, maximum tumor diameter, maximum solid component diameter and CTR. Our results suggest that STAS could be predicted with these radiological features with excellent sensitivity but inferior specificity.

\section{Acknowledgments}

Funding: None. 


\section{Footnote}

Reporting Checklist: The authors have completed the STROBE reporting checklist. Available at http://dx.doi. org/10.21037/jtd-20-1820

Data Sharing Statement: Available at http://dx.doi. org/10.21037/jtd-20-1820

Conflicts of Interest: All authors have completed the ICMJE uniform disclosure form (available at http://dx.doi. org/10.21037/jtd-20-1820). DL serves as an unpaid editorial board member of Fournal of Thoracic Disease from Nov 2016 to Oct 2020. The other authors have no conflicts of interest to declare.

Ethical Statement: The authors are accountable for all aspects of the work in ensuring that questions related to the accuracy or integrity of any part of the work are appropriately investigated and resolved. The study was conducted in accordance with the Declaration of Helsinki (as revised in 2013). The study was approved by the institutional ethics board of China-Japan Friendship Hospital (No. 2018-13-K08), and individual consent for this retrospective analysis was waived.

Open Access Statement: This is an Open Access article distributed in accordance with the Creative Commons Attribution-NonCommercial-NoDerivs 4.0 International License (CC BY-NC-ND 4.0), which permits the noncommercial replication and distribution of the article with the strict proviso that no changes or edits are made and the original work is properly cited (including links to both the formal publication through the relevant DOI and the license). See: https://creativecommons.org/licenses/by-nc-nd/4.0/.

\section{References}

1. Chen $\mathrm{W}$, Zheng R, Baade PD, et al. Cancer statistics in China, 2015. CA Cancer J Clin 2016;66:115-32.

2. Bray F, Ferlya J, Soerjomataram I, et al. Global cancer statistics 2018: GLOBOCAN estimates of incidence and mortality worldwide for 36 cancers in 185 countries. CA Cancer J Clin 2018;68:394-424.

3. National Lung Screening Trial Research Team, Aberle DR, Adams AM, et al. Reduced lung-cancer mortality with low-dose computed tomographic screening. N Engl J Med 2011;365:395-409.
4. Bongiolatti S, Corzani R, Borgianni S, et al. Long-term results after surgical treatment of the dominant lung adenocarcinoma associated with ground-glass opacities. J Thorac Dis 2018;10:4838-48.

5. Ginsberg RJ, Rubinstein LV. Randomized trial of lobectomy versus limited resection for T1 N0 non-small cell lung cancer. Lung Cancer Study Groups. Ann Thorac Surg 1995;60:615-22.

6. Suzuki K, Watanabe SI, Wakabayashi M, et al. A nonrandomized confirmatory phase III study of sublobar surgical resection for peripheral ground glass opacity dominant lung cancer defined with thoracic thin-section computed tomography (JCOG0804/WJOG4507L). J Clin Oncol 2017;35:abstr 8561.

7. Ye T, Deng L, Wang S, et al. Lung adenocarcinomas manifesting as radiological part-solid nodules define a special clinical subtype. J Thorac Oncol 2019;14:617-27.

8. Hattori A, Matsunaga T, Takamochi K, et al. Importance of ground glass opacity component in clinical stage IA radiologic invasive lung cancer. Ann Thorac Surg 2017;104:313-20.

9. Nakamura K, Saji H, Nakajima R, et al. A phase III randomized trial of lobectomy versus limited resection for small-sized peripheral non-small cell lung cancer (JCOG0802/WJOG4607L). Jpn J Clin Oncol 2010;40:271-4.

10. Blasberg JD, Pass HI, Donington JS. Sublobar resection: a movement from the Lung Cancer Study Group. J Thorac Oncol 2010;5:1583-93.

11. Travis WD, Brambilla E, Nicholson AG, et al. The 2015 World Health Organization Classification of lung tumors: impact of genetic, clinical and radiologic advances since the 2004 Classification. J Thorac Oncol 2015;10:1243-60.

12. Kadota K, Nitadori J, Sima CS, et al. Tumor spread through air spaces is an important pattern of invasion and impacts the frequency and location of recurrences after limited resection for small stage I lung adenocarcinomas. J Thorac Oncol 2015;10:806-14.

13. Masai K, Sakurai H, Sukeda A, et al. Prognostic impact of margin distance and tumor spread through air spaces in limited resection for primary lung Cancer, J Thorac Oncol 2017;12:1788-97.

14. Warth A, Muley T, Kossakowski CA, et al. Prognostic impact of intra-alveolar tumor spread in pulmonary adenocarcinoma, Am J Surg Pathol 2015;39:793-801.

15. Goldstraw P, Chansky K, Crowley J, et al. The IASLC Lung Cancer Staging Project: Proposals for Revision of the TNM Stage Groupings in the Forthcoming (Eighth) 
Edition of the TNM Classification for Lung Cancer. J Thorac Oncol 2016;11:39-51.

16. Zeng $W$, Zhang $W$, Zhang J, et al. Systematic review and meta-analysis of video-assisted thoracoscopic surgery segmentectomy versus lobectomy for stage I non-small cell lung cancer. World J Surg Oncol 2020;18:44.

17. Razi SS, Nguyen D, Villamizar N. Lobectomy does not confer survival advantage over segmentectomy for nonsmall cell lung cancer with unsuspected nodal disease. J Thorac Cardiovasc Surg 2020;159:2469-83.e4.

18. Winckelmans T, Decaluwé H, De Leyn P, et al. Segmentectomy or lobectomy for early-stage non-smallcell lung cancer: a systematic review and meta-analysis. Eur J Cardiothorac Surg 2020;57:1051-60.

19. NCCN Clinical Practice Guidelines in Oncology: NonSmall Cell Lung Cancer. [2020-3-2]. Available online: https://www.nccn.org/professionals/physician_gls/pdf/ nscl.pdf

20. Kadota K, Kushida Y, Kagawa S, et al. Limited resection is associated with a higher risk of locoregional recurrence than lobectomy in stage I lung adenocarcinoma with tumor spread through air spaces. Am J Surg Pathol 2019;43:1033-41.

21. Dai C, Xie H, Su H, et al. Tumor spread through air spaces (STAS): a call for more evidence. J Thorac Dis 2017,9:E951-3.

22. Toyokawa G, Yamada Y, Tagawa T, et al. Significance of

Cite this article as: Zhang Z, Liu Z, Feng H, Xiao F, Shao W, Liang C, Sun H, Gu X, Liu D. Predictive value of radiological features on spread through air space in stage cIA lung adenocarcinoma. J Thorac Dis 2020;12(11):6494-6504. doi: $10.21037 /$ jtd-20-1820 spread through air spaces in resected pathological stage I lung adenocarcinoma. Ann Thorac Surg 2018;105:1655-63.

23. Lee JS, Kim EK, Kim M, et al. Genetic and clinicopathologic characteristics of lung adenocarcinoma with tumor spread through air spaces. Lung Cancer 2018;123:121-6.

24. Shiono S, Yanagawa N. Spread through air spaces is a predictive factor of recurrence and a prognostic factor in stage I lung adenocarcinoma. Interact Cardiovasc Thorac Surg 2016;23:567-72.

25. Kim SK, Kim TJ, Chung MJ, et al. Lung adenocarcinoma: CT features associated with spread through air spaces. Radiology 2018;289:831-40.

26. de Margerie-Mellon C, Onken A, Heidinger BH, et al. CT manifestations of tumor spread through airspaces in pulmonary adenocarcinomas presenting as subsolid nodules. J Thorac Imaging 2018;33:402-8.

27. Kameda K, Lu S, Eguchi T, et al. Can tumor spread through air spaces (STAS) in lung denocarcinomas be predicted pre-and intraoperatively? J Thorac Oncol 2017;12:S4112.

28. Pyo JS, Park MJ, Kang DW. The clinicopathological significance of micropapillary pattern in colorectal cancers. Hum Pathol 2018;77:159-65.

29. Pyo JS, Kim JH. Clinicopathological significance of micropapillary pattern in lung adenocarcinoma. Pathol Oncol Res 2018;24:547-55. 